\title{
Differential expression of COX-2 in osteoarthritis and rheumatoid arthritis
}

\author{
H.W. Fan', G.Y. Liu', C.F. Zhao', X.F. Li' ${ }^{1}$ and X.Y. Yang' ${ }^{2}$ \\ ${ }^{1}$ Department of Orthopedic, China-Japan Union Hospital of Jilin University, \\ Changchun Jilin, China \\ ${ }^{2}$ The Second Hospital of Jilin University, Orthopaedics Hospital, Changchun Jilin, \\ China \\ Corresponding author: X.Y. Yang \\ E-mail: Xiaoyuchina12@163.com \\ Genet. Mol. Res. 14 (4): 12872-12879 (2015) \\ Received May 2, 2015 \\ Accepted July 2, 2015 \\ Published October 21, 2015 \\ DOI http://dx.doi.org/10.4238/2015.October.21.7
}

ABSTRACT. In this study, we investigated the differential expression profiles of cyclooxygenase-2 (COX-2) mRNA and proteins in osteoarthritis $(\mathrm{OA})$ and rheumatoid arthritis (RA) patients to elucidate the role of COX-2 expression in the pathogenesis and development of these diseases and to provide novel drug targets for treating arthritis. A total of 60 patients who received arthroscopic surgeries for treating $\mathrm{OA}(\mathrm{N}=30)$ or $\mathrm{RA}(\mathrm{N}=30)$ were examined. Fifteen normal synovial tissue samples were included as the control group. Fibroblastic synovial cells in all samples were cultured in vitro and COX-2 mRNA, protein expression levels, and COX-2 levels were detected in synovial fluids by real-time polymerase chain reaction, western blotting, and enzyme-linked immunosorbent assay methods, respectively. The mRNA level of COX-2 was significantly elevated in synovial cells from $O A$ and RA patients compared to that in control samples $(P<0.05)$. COX2 mRNA level was significantly higher in synovial cells from OA patients than in those from RA patients $(P<0.05)$. Consistent results were obtained for COX-2 protein expression levels from patients' synovial samples. In synovial fluids, OA $(P<0.05)$, but not RA $(P>0.05)$, patients showed significantly higher COX-2 levels compared to the control group. Elevated 
synovial COX-2 expression facilitates the pathogenesis of OA and RA, and thus this index reflects the condition of these 2 diseases.

Key words: Cyclooxygenase-2; Osteoarthritis; Rheumatoid arthritis; Synovial tissues

\section{INTRODUCTION}

As a degenerative articular cartilage disease, osteoarthritis (OA) affects the normal activity of approximately one-third of the population worldwide. The incidence of OA continues to increase as the population ages; this condition is also known as degenerative arthritis or senescent arthritis (Rahimzadeh et al., 2014). In China, approximately $60 \%$ of aged people suffer from OA, which severely affects their quality of life (Grønhaug et al., 2014). OA is induced by various factors including the age, joint strain, trauma, and weight gain, all of which lead to articular cartilage damage and further cause reactive hyperplasia of articular edge and subchondral bones. Thus, this disease induces manifestations such as chronic progressive joint swelling, pains, stiffness, and even joint deformities (Bao et al., 2014; Sowa et al., 2014; Yang et al., 2014).

Rheumatoid arthritis (RA) is an auto-immune disease with an increasing incidence in aging people, although it can occur at any age. Little is known regarding the pathogenesis of RA, which is a systematic disease involving chronic inflammation of synovial tissues (Hirata and Tanaka, 2014). RA is mainly manifested as invasive and symmetric arthritis affecting small articular joints such as those in the hands and feet, and can lead to joint deformities and dysfunctions; RA is also accompanied by multiple extra-articular organ diseases and positive serum rheumatoid factors (Giangreco and Cutolo, 2014; Lattanzi et al., 2014).

As a necessary and rate-limiting enzyme in the biosynthesis of prostaglandin, cyclooxygenase (COX) has 2 isoforms, including COX-1 and COX-2, which share homology as high as 60\% but have distinct functions (Rao et al., 2014; Stepensky and Rimon, 2014). Studies have demonstrated the physiological role of COX-1 in the body's normal metabolism and protection by the release of prostaglandin, while COX-2 has been recognized as an inducible or pathological enzyme that can be induced by various chemical/physical injuries or biological factors. COX-2 also participates in the inflammatory response by facilitating prostaglandin synthesis (Lee et al., 2014b; Shimomura et al., 2014). The pathogenesis of OA or RA is not well-understood, although abnormal expression of certain genes or proteins has been found to be correlated with the occurrence of these diseases to some degree. Among these related genes, COX-2 plays an important role in articular cartilage disease and studies have reported that the specific expression of COX-2 not only affected normal cellular functions but also facilitated the inflammatory cytokine-induced metabolic imbalance of cartilage proteoglycans, thereby irreversibly advancing the arthritis (Huang and Huang, 2014; Lee et al., 2014a). Therefore, we investigated the differential expression of COX-2 mRNA and proteins in synovial cells and joint fluids from OA and RA patients to determine the role of COX-2 expression in the pathogenesis of these 2 diseases. Our results may provide novel drug targets for treating various types of arthritis.

\section{MATERIAL AND METHODS}

\section{Research subjects}

A total of 60 OA or RA patients who received arthroscopic surgeries between January 
2010 and December 2013 in our hospital were recruited for this study. There were 30 cases of OA (17 males, 13 females, 41-78 years old, average age of $56.4 \pm 8.2$ years) and 30 RA patients (12 males, 18 females, 45-62 years old, average age of $52.1 \pm 6.7$ years). Additionally, another 15 normal synovial tissue samples were included in the control group from knee joint trauma patients (including 8 meniscus injuries and 7 cases of cruciate ligament injuries; 6 males and 9 females, 3865 years old, average age of $53.1 \pm 5.6$ years). Both OA and RA patients were diagnosed based on clinical symptoms, pathological examinations, X-ray films, and rheumatoid factor assays. Included patients had not received related medication previously or taken other medicine within 2 weeks. Patients with active gastrointestinal ulcers, kidney, liver disease, blood coagulation dysfunctions, or complicated with heart or brain vascular disease were excluded from this study, which also excluded pregnant or lactating women. There were no statistically significant differences in the general information of patients, such as sex or age, across all research subjects $(P>0.05)$.

The study protocol was approved by the Research Ethics Committee of our hospital, and all patients provided informed consent before study commencement.

\section{Separation and culture of articular synovial cells}

Synovial tissue samples collected during the surgery were rinsed in $0.01 \mathrm{M}$ sterilized

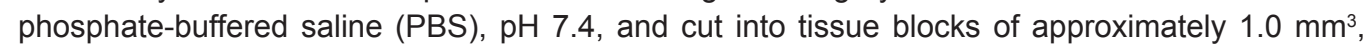
which were then centrifuged at $1000 \mathrm{~g}$ for $5 \mathrm{~min}$ and cultured using the adhesion method with $20 \%$ fetal bovine serum (Hyclone, Logan, UT, USA) and $80 \%$ high-glucose DMEM medium (Hyclone) containing $100 \mathrm{U} / \mathrm{mL}$ penicillin and streptomycin (Hyclone). Tissue blocks were laid with $5 \mathrm{~mm}$ and cultured in an inverted culture tube at $37^{\circ} \mathrm{C}$ in an incubation chamber (Thermo Scientific, Waltham, MA, USA) with $5 \% \mathrm{CO}_{2}$. The culture medium was changed after 3-4 days based on cell growth. Cells after primary culture were passaged when the fusion reached $80-90 \%$.

\section{Collection of joint fluid samples}

Two milliliters joint fluid samples collected during surgery were diluted 1:1000 in sterilized PBS and were stored at $4^{\circ} \mathrm{C}$ until use.

\section{Assay of COX-2 mRNA levels by real-time polymerase chain reaction (PCR)}

RNA from cultured synovial cells in the log phase was extracted using Trizol reagent (Invitrogen, Carlsbad, CA, USA). Total RNA purity and concentration was measured using a UV spectrophotometer. An in vitro reverse transcription was performed using a reaction kit (Invitrogen) with the total RNA as the template. Specific primers (COX-2-F: 5'-TCGCT GTGCC TGATG ATTCC-3'; COX-2-R: 5'-CTTAT GATCT CGTTT CCGTC-3'; amplified length of 196 base pairs; GAPDH-F: 5'-TACCA GTAGC TCTGG GTTG-3'; GAPDH-R: 5'- GACTAATATG TCTGG TCCGG A-3'; amplified length of 205 base pairs) were designed and synthesized by Shanghai Shenggong Biotech (Shanghai, China) to produce target cDNA. The real-time PCR assay was performed on target genes using GAPDH as an internal reference. PCR parameters were as follows: $50^{\circ} \mathrm{C}$ for $1 \mathrm{~min}$, followed by 35 cycles of $95^{\circ} \mathrm{C}$ for $30 \mathrm{~s}$ for denaturation, $56^{\circ} \mathrm{C} 30 \mathrm{~s}$ for annealing, and $72^{\circ} \mathrm{C} 30 \mathrm{~s}$ for extension. Raw data was collected from the fluorescent quantitative PCR reactor and the sample's initial cycling numbers (CT values) were determined. Additionally, CT values of standard samples were used to plot the standard curve, which was further quantitatively analyzed by the $2-^{\mathrm{DDCt}}$ method. 


\section{Assay of COX-2 proteins using western blotting}

When the cell fusion rate approached $80 \%$ or above, lysis buffer was added to process the cells for $30 \mathrm{~min}$ on ice followed by ultrasound lysis of cells, which were centrifuged at 10,000 $g$ and $4^{\circ} \mathrm{C}$ for $15 \mathrm{~min}$. The supernatant was transferred to a new tube, protein concentration was determined, and samples were stored at $-20^{\circ} \mathrm{C}$ until western blotting. Protein elusion buffers were mixed with the cell lysate, which was denatured at $95^{\circ} \mathrm{C}$ for $5 \mathrm{~min}$. Proteins were separated by $10 \%$ sodium dodecyl sulfate-polyacrylamide gel electrophoresis, and bands were transferred to a polyvinylidene fluoride membrane (Pall Corporation, Port Washington, NY, USA), which was blocked for non-specific binding using 5\% defatted milk powders for $2 \mathrm{~h}$. Primary antibodies including antiCOX2 rabbit IgG (1:1000 dilution, Cell Signaling Technology, Danvers, MA, USA) was added for overnight incubation followed by washing with PBS containing Tween 20 (PBST). Mouse anti-rabbit IgG conjugated to horseradish peroxidase (1:2000 dilution, Cell Signaling Technology) was then incubated with the membrane at room temperature for $30 \mathrm{~min}$ in the dark. After washing with PBST, enhanced electrochemiluminescence chromogenic reagents (Amersham Biosciences, Amersham, UK) were used to develop the membrane for $1 \mathrm{~min}$, followed by exposure under X-ray. X-ray images were scanned and analyzed using the Bio-Rad image analysis system (Hercules, CA, USA), while the optical density of bands was measured using Quantity One software (Bio-Rad). Anti-actin antibody was also used as an internal reference. Each experiment was repeated at least 4 times.

\section{Expression of COX-2 in joint fluids by enzyme-linked immunosorbent assay}

We used an enzyme-linked immunosorbent assay kit (Shanghai Beyotime Biotech, Shanghai, China) to quantify the expression level of COX-2 proteins in joint fluids from all groups following the manual instructions. Briefly, $50 \mathrm{~mL}$ serially diluted standard samples were to 96 -well plates in triplicate to generate the standard curve. Additionally, $50 \mathrm{~mL}$ test samples were also added to 96-well plates in triplicate. After washing with PBST, enzyme-labeled antibody was added into each well except for the blank well. After gentle mixing and incubation at $37^{\circ} \mathrm{C}$ for $30 \mathrm{~min}$, PBST was used to wash the plates several times. The substrates were then added for 10-min development in the dark at $37^{\circ} \mathrm{C}$. Absorption at $450 \mathrm{~nm}\left(\mathrm{OD}_{450}\right)$ was measured using a microplate reader using the blank well as the reference point. Finally, sample concentrations were calculated based on the standard curve.

\section{Statistics}

SPSS 16.0 software package was used to process all collected data (SPSS, Inc., Chicago, IL, USA), of which quantitative data were presented as the mean \pm SD, unless otherwise specified. One-way analysis of variance was used to compare mean values among multiple groups while Student $t$-test was applied to compare means between 2 groups. Statistical significance was defined when $\mathrm{P}<0.05$.

\section{RESULTS}

\section{Expression of COX-2 mRNA in OA and RA synovial tissues}

The expressional profiles of COX-2 mRNA were analyzed using real-time PCR in synovial 
tissue samples from both OA and RA patients. The results showed significantly elevated COX-2 mRNA in synovial cells in OA and RA patients compared with those from healthy tissues (Figure $1, P<0.05)$. Further between-group comparison revealed significantly higher mRNA levels in OA synovial cells than in RA samples $(P<0.05)$.

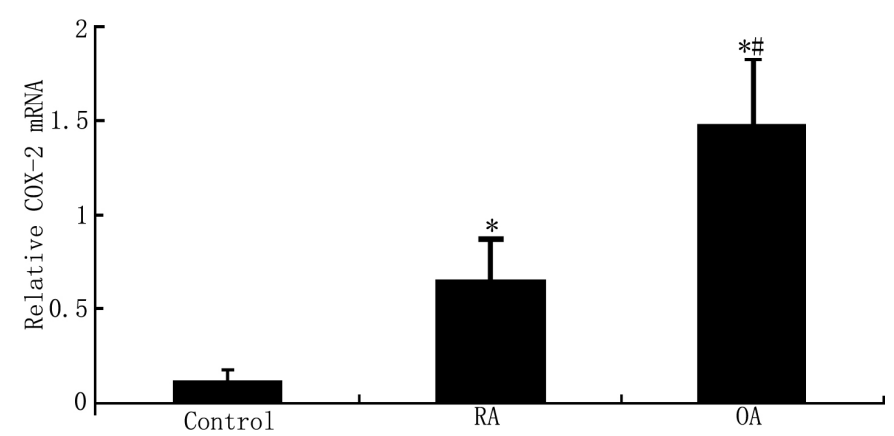

Figure 1. COX-2 mRNA expression in OA and RA patients. ${ }^{*} \mathrm{P}<0.05$ compared to control group; $\mathrm{F}<0.05$ compared to RA group.

\section{COX-2 protein expression in OA and RA patients}

We further analyzed the protein expression level of COX-2 in synovial cells from both $\mathrm{OA}$ and RA patients using western blotting. The results showed consistent patterns with the results for mRNA levels, and OA or RA synovial cells contained significantly higher COX-2 protein levels, which were relatively lower in healthy tissues (Figure 2, $\mathrm{P}<0.05$ ). Moreover, OA patients had significantly elevated COX-2 proteins in their synovial tissues compared with levels in RA patients (Figure 2, $\mathrm{P}<0.05$ ). Expressional profiles of both mRNA and protein of COX-2 in arthritis patients' synovial tissues were consistent, strongly supporting the role of COX-2 up-regulation in the pathogenesis of OA and RA.

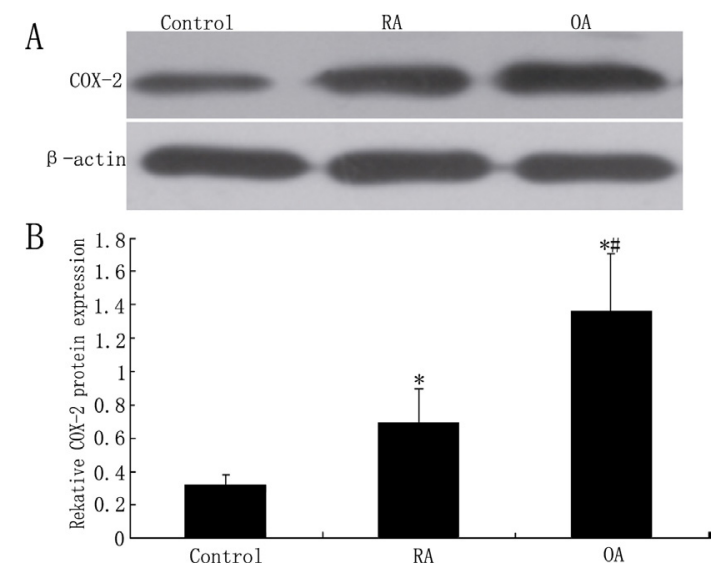

Figure 2. COX-2 protein expression in synovial cells. A. Representative western blotting bands using anti-COX-2 and anti-actin as an internal reference. Both RA and OA samples had higher intensity of bands compared to control sample. B. Quantitative analysis of results in (A) by the relative expression level of COX-2 using $\beta$-actin as the reference. ${ }^{*} P<$ 0.05 compared to control group; ${ }^{P} \mathrm{P}<0.05$ compared to RA group. 


\section{COX-2 expression in joint fluids from OA and RA patients}

The expressional profiles of COX-2 in joint fluids from all groups were further analyzed using an enzyme-linked immunosorbent assay technique. The results showed significantly higher COX-2 levels in OA joint fluids (Figure 3, $P<0.05)$, but not in RA samples $(P>0.05)$, compared with the control group. The concurrent increase in COX-2 expression in both synovial tissues and joint fluids in OA patients suggested that COX-2 is a potent trophic factor in the pathogenesis of OA. The occurrence of RA, however, may involve the differential expression of COX-2 in synovial cells.

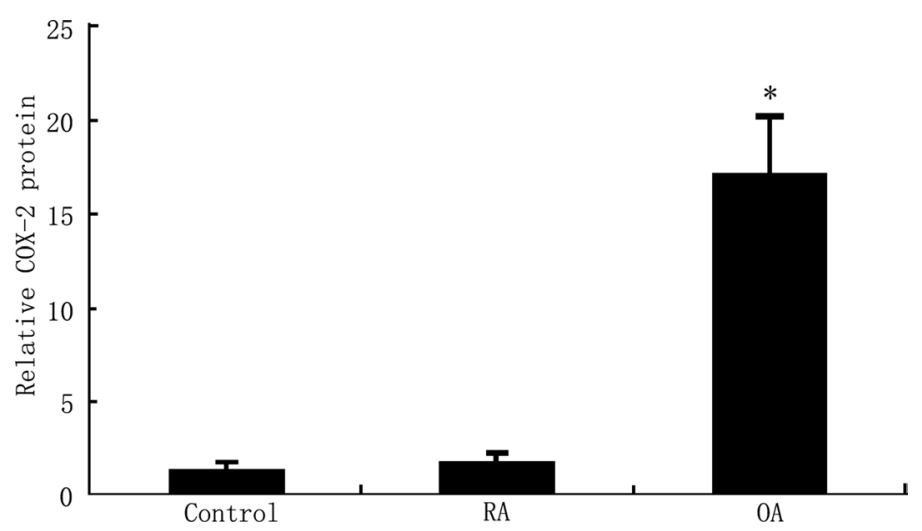

Figure 3. Differential expression of COX-2 in joint fluids from RA and OA patients. ${ }^{*} P<0.05$ compared to control group.

\section{DISCUSSION}

Both OA and RA can cause joint swelling and dysfunction to various extents, and even causing function loss and disabilities of patients. The incidences of OA and RA have increased and become major health issues for aging people (Sadeghi-Aliabadi et al., 2013; Choi et al., 2014). Although complex pathogenic mechanisms are involved in these diseases, articular synovial tissues are thought to play an important role, as both intra-articular cartilage lesions and secondary inflammatory lesions caused by synovial inflammations can facilitate the occurrence and advancement of OA and RA, further aggravating the clinical symptoms (Kumagai et al., 2013; Saravanan et al., 2014). Hyperplasia or even fibrosis of synovial tissues can stimulate the occurrence of large numbers of inflammatory cells as well as the section of pro-inflammatory factors, making this a critical factor in the pathogenesis of OA and RA (Yoon et al., 2013).

Under normal physiological conditions, COX-2 is expressed at low levels. Under the induction of pro-inflammatory factors from inflammation sites, however, COX-2 can be abundantly released from various cells including monocytes, macrophages, synovial cells, fibroblasts, and endothelial cells, all of which participate in the inflammatory process and cause body damage (Wu et al., 2013). Multiple pathological and physiological functions of COX-2 have been identified, and numerous studies have examined the pathogenesis of OA and RA. In vitro studies have consistently observed the proliferation of articular synovial cells with the induction of inflammatory factors. The introduction of a COX-2 inhibitor significantly suppresses the proliferation and differentiation of articular synovial cells, thereby depressing synovial inflammation and preventing joint injury. For example, down-regulation of tumor necrotic factor a can inactivate the transcriptional activity of 
nuclear factor-kB, which inhibits downstream COX-2 expression and thus effectively manages the advancement of inflammation (Haseeb et al., 2013; Yu and Kim, 2013). These studies have demonstrated the presence and expression of COX-2 in articular synovial cells and have suggested that a relationship exists between COX-2 and arthritis. However, the differential expression of COX-2 in OA and RA patients, as well as whether COX-2 can function as a drug target in disease prevention or treatment has not been reported. This study revealed relatively lower expression of COX-2 in terms of both mRNA and protein in healthy subjects, but significantly elevated expression in OA and RA patients' articular synovial cells (Figure 1 and 2). Furthermore, OA patients showed even higher COX2 mRNA and protein levels when compared to RA patients (Figure 1 and 2). Collectively, these results suggest the potency of synovial COX-2 in facilitating the pathogenesis of OA and RA. In contrast, COX-2 expression level was elevated in both synovial tissues (Figure 2) and joint fluids (Figure 3) of OA patients, making COX-2 a potent trophic factor of OA. The occurrence of RA, however, may mainly function with the differential expression of COX-2 in synovial cells.

In summary, elevated expression of COX-2, particularly in synovial cells can facilitate the occurrence of both OA and RA. Therefore, COX-2 is an important index and potential drug target for these diseases. It may be possible to apply specific COX-2 inhibitors to suppress the progression of arthritis.

\section{Conflicts of interest}

The authors declare no conflict of interest.

\section{ACKNOWLEDGMENTS}

We thank the anonymous reviewers for reviewing this manuscript.

\section{REFERENCES}

Bao JP, Jiang LF, Chen WP, Hu PF, et al. (2014). Expression of vaspin in the joint and the levels in the serum and synovial fluid of patients with osteoarthritis. Int. J. Clin. Exp. Med. 7: 3447-3453.

Choi YJ, Lee WS, Lee EG, Sung MS, et al. (2014). Sulforaphane inhibits IL-1 $\beta$-induced proliferation of rheumatoid arthritis synovial fibroblasts and the production of MMPs, COX-2, and PGE2. Inflammation 37: 1496-1503.

Giangreco D and Cutolo M (2014). Morning glucocorticoids versus night glucocorticoids: the role of low-dose glucocorticoid chronotherapy in rheumatoid arthritis. J. Clin. Rheumatol. 20: 437-439.

Grønhaug G, Østerås N and Hagen K (2014). Quality of hip and knee osteoarthritis management in primary health care in a Norwegian county: a cross-sectional survey. BMC Health Serv. Res. 14: 598.

Haseeb A, Chen D and Haqqi TM (2013). Delphinidin inhibits IL-1 $\beta$-induced activation of NF-kB by modulating the phosphorylation of IRAK-1(Ser376) in human articular chondrocytes. Rheumatology (Oxford) 52: 998-1008.

Hirata S and Tanaka Y (2014). Combination therapy for early rheumatoid arthritis: a treatment holiday perspective. Expert Rev. Clin. Pharmacol. 8: 115-122.

Huang QC and Huang RY (2014). The cyclooxygenase-2/thromboxane A2 pathway: a bridge from rheumatoid arthritis to lung cancer? Cancer Lett. 354: 28-32.

Kumagai K, Kubo M, Imai S, Toyoda F, et al. (2013). The COX-2 selective blocker etodolac inhibits TNFalpha-induced apoptosis in isolated rabbit articular chondrocytes. Int. J. Mol. Sci. 14: 19705-19715.

Lattanzi S, Cagnetti C, Di Bella P, Scarpelli M, et al. (2014). Leptomeningeal inflammation in rheumatoid arthritis. Neurol. Neuroimmunol. Neuroinflamm. 1: e43.

Lee KH, Abas F, Mohamed Alitheen NB, Shaari K, et al. (2014a). Chemopreventive effects of a curcumin-like diarylpentanoid [2,6-bis(2,5-dimethoxybenzylidene)cyclohexanone] in cellular targets of rheumatoid arthritis in vitro. Int. J. Rheum. Dis. Epub ahead of print. 
Lee WS, Lim JH, Sung MS, Lee EG, et al. (2014b). Ethyl acetate fraction from Angelica sinensis inhibits IL-1beta-induced rheumatoid synovial fibroblast proliferation and COX-2, PGE2, and MMPs production. Biol. Res. 47: 41.

Rahimzadeh P, Imani F, Faiz SH, Entezary SR, et al. (2014). Investigation the efficacy of intra-articular prolotherapy with erythropoietin and dextrose and intra-articular pulsed radiofrequency on pain level reduction and range of motion improvement in primary osteoarthritis of knee. J. Res. Med. Sci. 19: 696-702.

Rao SR, Balaji TM, Prakash PS and Lavu V (2014). Elevated levels of cyclooxygenase 1 and 2 in human cyclosporine induced gingival overgrowth. Prostaglandins Other Lipid Mediat. 113-115: 69-74.

Sadeghi-Aliabadi H, Aliasgharluo M, Fattahi A, Mirian M, et al. (2013). In vitro cytotoxic evaluation of some synthesized COX-2 inhibitor derivatives against a panel of human cancer cell lines. Res. Pharm. Sci. 8: 298-303.

Saravanan S, Hairul Islam VI, Prakash Babu N, Pandikumar P, et al. (2014). Swertiamarin attenuates inflammation mediators via modulating NF-kB/l kB and JAK2/STAT3 transcription factors in adjuvant induced arthritis. Eur. J. Pharm. Sci. 56: 70-86.

Shimomura K, Kanamoto T, Kita K, Akamine Y, et al. (2014). Cyclic compressive loading on 3D tissue of human synovial fibroblasts upregulates prostaglandin E2 via COX-2 production without IL-1 $\beta$ and TNF- $\alpha$. Bone Joint Res. 3: 280-288.

Sowa GA, Perera S, Bechara B, Agarwal V, et al. (2014). Associations between serum biomarkers and pain and pain-related function in older adults with low back pain: a pilot study. J. Am. Geriatr. Soc. 62: 2047-2055.

Stepensky D and Rimon G (2014). Competition between low-dose aspirin and other NSAIDs for COX-1 binding and its clinical consequences for the drugs' antiplatelet effects. Expert Opin. Drug Metab. Toxicol. 11: 41-52.

Wu MY, Yang RS, Lin TH, Tang CH, et al. (2013). Enhancement of PLGF production by 15-(S)-HETE via PI3K-Akt, NF-kB and COX-2 pathways in rheumatoid arthritis synovial fibroblast. Eur. J. Pharmacol. 714: 388-396.

Yang Y, Tien HY, Kumar KK, Chen S, et al. (2014). Ligament reconstruction with tendon interposition arthroplasty for first carpometacarpal joint osteoarthritis. Chin. Med. J. (Engl.) 127: 3921-3925.

Yoon HY, Lee EG, Lee H, Cho IJ, et al. (2013). Kaempferol inhibits IL-1 $\beta$-induced proliferation of rheumatoid arthritis synovial fibroblasts and the production of COX-2, PGE2 and MMPs. Int. J. Mol. Med. 32: 971-977.

Yu SM and Kim SJ (2013). Production of reactive oxygen species by withaferin A causes loss of type collagen expression and COX-2 expression through the PI3K/Akt, p38, and JNK pathways in rabbit articular chondrocytes. Exp. Cell Res. 319: $2822-2834$ 